\title{
Current research status of forecast criteria for landslide monitoring and forecasting system in China
}

\author{
Xiangjun Chen \\ Shijiazhuang Tiedao University, Shijiazhuang, China,050043 \\ chenxiangjun@stdu.edu.cn,
}

\begin{abstract}
Keywords: Landslide, forecast, early-warning, forecast criterion
\end{abstract}
Abstract. The threat to people's lives and property safety posed by landslide disasters has aroused great concern of the researchers in China. Landslide monitoring and forecasting is an important means to prevent and mitigate landslide disasters. Determination of the forecast criteria is a key technology of the landslide monitoring and early-warning systems. Some forecast models taking single parameter as forecast criteria and some other forecast models using the comprehensive forecast criteria are introduced. The multifactor comprehensive forecast model based on geological analysis, simulation analysis, and intelligent information technology can fully reflect the personality characteristics and evolution process of landslide, so it can ensure maximum effectiveness of the monitoring and early-warning.

\section{Introduction}

China is a geological disaster especially landslide disaster-prone countries. According to the monitoring data from China Institute of Geo-Environment Monitoring, thousands of times landslide disaster, accounting for over $70 \%$ of all geological disasters, happened nationwide in recent years. Large scale landslide disaster seriously endangers people's lives and property. For example, at 22:50 on November 13, 2015, landslides took place at Lishui City, Zhejiang Province. Collapse landslide volume was more than 30 million cubic meters, 27 houses were buried and 38 people were killed. At 20:30 on August 27,2014 approximately, landslide took place at Fuquan, Guizhou Province. Two groups of villagers' house were buried and 23 people were killed.

These typical catastrophic events illustrate that landslide disasters influence widely and have diverse endangering forms. Fully recognizing the seriousness of the threat to people's lives and property safety posed by landslide disasters in China, in order to avoid and mitigate the harm the landslides caused, people have been studying how to take some measures to predict and prevent landslides. Landslide monitoring and forecasting is an important means to prevent and mitigate landslide disasters. The monitoring work is only a means, scientific and effective prediction is the ultimate goal. The changes of certain characteristics during the landslide development process are monitored. The time, space and scale of landslide can be forecasted timely by analyzing the variation monitored. According the results the forecasting, some effective measures can be taken to reduce the harm caused by the landslide timely.

Through more than 10 years of research and monitoring work, a major catastrophic landslide taking place at Xintan Town, Zigui County of Hubei Province at 3:45 on June 12, 1985was predicted successfully by Chinese scientists. 12 deformation observation points were set at the region for long-term monitoring from 1977. Monitoring results and field survey data shew that the region began to appear abnorma in 1983. Untill to June 11, 1985, when the field investigation and displacement monitoring data very strongly suggested that the upcoming big slide would happen. Monitoring staff issued a danger in an emergency under the condition of so obvious portent. Just over 11 hours, then the landslide occurred. It is a devastating geological disaster, which the volume of the landslide is about 3,000 million cubic meters. Due to the forecast timely, the 1371 residents in the Xintan Town were no casualties. The decisive and effective evacuation measures were taken to reduce economic losses and casualties. 


\section{Importance of forecast criteria in forecasting and early-warning systems}

Connotation of landslide prediction is very wide, broadly including landslide space prediction, time forecasting and risk prediction. The majority of single landslides are unstable time prediction. According to the time scale, forecast can be broadly divided into three stages, which are long-term forecasting, short-term forecasting and temporary slip prediction. For different forecasting scales, data and information relied are different, so the prediction accuracy and the objectives are different. For example, long-term forecast generally takes geological characteristics, long-term rainfall patterns, landslide stability factor, failure probabilityand other parameters as the basis. The main goal is to forecast the development trend of landslide deformation and likelihood of occurrence. The temporary slip forecasts are mostly based on landslide's specific unstable time gotten by quantitative landslide displacement monitoring data and other parameters. Generally speaking, landslide deformation prediction belongs to short-term and long-term forecasting category. Landslide unstable time forecast belongs to the temporary slip prediction category. However, instability and failure is the result of landslide deformation progressive development, the two above are often studied together.

Landslide disaster prediction and early-warning has been difficulty and hotspot of landslide research. Determination of the forecast criteria is a key technology of the landslide monitoring and early-warning systems. Reasonable forecasting criterion can make the monitoring and warning system give play to the greatest benefit. It not only can avoid unnecessary panic and waste caused by false forecasting, but also can avoid the enormous risks and destruction by omission forecasting. Forecasting criterion determining is also a core technology of the monitoring and warning system. All monitoring data must be analyzed and compared with the forecasting criterion firstly. Then the comparing results can be used to service for the forecasting and warning. So the method for determining prediction criterion directly affects the design and implementation of monitoring programs. In recent decades, many geological experts form different countries dedicated to the study, but has yet to get satisfactory results. Given the importance of the determining method for forecasting criterion, " Plan for the Prevention and Control of Geological Disasters" was issued by China's State Council. It stated clearly that the research of geological disaster monitoring and prediction criteria should be gotten on the basis of a long-time accumulating data. Based on this, it can promote high-tech monitoring technologies gradual transition from the experimental stage to the practical stage.

\section{Current research status of single parameter forecast criteria}

Landslide is a process influenced by many factors. So its forecasting is very difficult. Early research on landslide is focused mainly on the qualitative trend judge of the landslide instability in China. The research is mainly based on the deformation and failure phenomenon, unstable macro precursor, simple displacement monitoring and relevant experience in engineering geology. 1980 years later, the research is more focus on landslide quantitative forecasting methods. Many landslide prediction models use a single parameter as forecasting criterion are proposed. Since the 1990s, affected by scientific development of system theory and nonlinear theory, nonlinear theory is introduced to study the problem of landslide forecasting by a number of scholars. The introduction of the nonlinear theory improves the method for determining prediction criterion of single parameter. Landslide prediction models which take single parameter as forecast criteria include the rainfall parameters forecasting method[1,2], displacement parameters forecasting method[3,4], crack observation forecasting method[5], acoustic emission parameters forecasting method[6], surface angle changes prediction method [7] and so on. The precipitation forecast parameters method and displacement parameters prediction method are the two most important methods. They obtain the sustained attention of the researchers and get great progress. 
According to statistics, about half of the Chinese mainland's major catastrophic landslide directly trigger by heavy rainfall since the 1980s. Therefore, research on the impact mechanism of rainfall on landslide is very necessary. In order to reduce or eradicate rainfall landslide risks, appropriate disaster prevention measures must be adopted and effective disaster warning mechanism must be established. Dayong Liu analyzed the relationship between landslide and rainfall in Fushun West Open Pit, and proposed a method for the Prediction of such landslides based on Rainfall and rainfall intensity factor [1]. Depth study on rainfall parameters forecasting method was carried out by Zhilei Wang[2] based on analysis of the relationship among rainfall, groundwater level and surface displacement. A landslide forecast System based on rainfall monitoring is established to assist the traditional landslide forecast system based on surface displacement. Rainfall monitoring is easier to operate than displacement monitoring. Therefore rainfall monitoring is particularly suitable for a wide range of regional landslide monitoring. Of course, it is also suitable for individual important landslide monitoring.

Displacement parameters prediction method can achieve quantitative temporary landslide warning and forecasting by researching time series data of displacement monitoring changes to forecas the slope deformation trends. It is particularly suited for the individual important landslide monitoring. Based on a large number of landslide deformation monitoring data, the change laws of accumulative displacement, deformation rate and acceleration were studied by Qiang $\mathrm{Xu}$ [3]; It is found that the changes of acceleration performance have different characteristics with displacement and deformation rate. the acceleration before and after the landslide shows completely different characteristics. Aiming at the above characteristics, the early-warning prediction indicator for critical landslide sliding is proposed based on the acceleration. According to the indicator, automatic early-warning prediction of critical landslide sliding is expected to achieve. Taking Jinping Hydropower Station for example, five landslides that are most similar with the slope at left bank of Jinping Hydropower Station were studied by Cong Li[4]. Based on deep analysis of deformation and failure process of these similar slopes, some factors that affect the slope warning criterion is determined, namely the failure mode, sliding face angle, slip surface type, structure of rock slope, slope angle, rainfall, groundwater, reservoir storage, excavation, blasting, earthquake acceleration, etc., A fuzzy comprehensive assessment model based on these 11 factors is established to revise the displacement rate threshold.

Single parameter prediction method involves only one or a class of parameters. This method has several features such as easy-operating on-site monitoring equipment, easy understanding monitoring data, simple data processing process, and so on. It is suitable for area where the geological environment pattern is relatively simple. However, due to simplification of the landslide factors, the accuracy and practicality of most of the models is limited. At the same time, the universality is not strong.

\section{Current research status of the Comprehensive forecast criteria}

The landslides have strong personality characteristics. Their evolutionary behavior of the deformation is closely related to the environment conditions and the geological structure of the slope. Single parameter criterion is difficult to reflect the combined effects of landslide various factors. In addition, it may cause false alarms or underreporting. Since the 1990s, many researchers began to realize that landslide forecasting study from a pure methodological point of view, which mainly rely on mathematical deduction to get the forecasting model, can not truly reveal the dynamic process of landslide deformation and its accuracy has is a certain gap with practical requirements. Researchers began to emphasize the system characteristics of the landslide, the dynamic comprehensive forecasting methods and geological mechanism research of landslide. On this basis, they developed a number of landslide comprehensive prediction model and some predictions ideas with practical value, such as comprehensive forecasting system, real-time tracking forecasts, etc[8,9]. Especially in the last decade, people gradually pay attention to the comprehensive prediction criterion study of landslide, some researchers have made some useful exploration and research in this area[10,11]. 
The Comprehensive forecasts criteria can not be achieved without the support from the three-dimensional monitoring network with real-time monitoring, real-time transmission and real-time data processing functions. In recent years, with the development of the real-time monitoring systems, some real-time, automatic and remote monitoring systems are established for landslide monitoring and prediction. Based on the mechanical principle of interaction among landslide body, landslide bed and monitoring anchor cable of slope, the mechanical principle of relative movement between landslide body and landslide bed was proposed in Manchao He's research[12]. The multi-factor monitoring is transformed into single landslide mechanical mointoring and the relationship between sliding force of slope and pretightening force is provided. According to the physical model experiment of landslide, the stress will change continually inside the landslide mass before the occurrence of landslide. When the sliding force is greater than the anti-slideing force, the deformation and landslide will take place, which means the change of stess will be ahead of displacement. Congsequently, monitoring the alteration of stress in the sliding mass is better than that of the displacement. Based on the above principle and experiment, the system of real-time remote monitoring and forecasting of landslide was developed by Manchao He's research team, which can realize the real-time remote monitoring of perturbation. According to the field application to opencast mines slope, this new technology successfully implements monitoring and forecasting for stable condition of slopes.

Depth study on Multi-parameter prediction model for regional landslide monitoring and forecasting was carried out by Mingsheng wen[13]. A real-time automatic monitoring network including more than 98 monitoring instruments, which can monitor rain, moisture content, displacement and other kinds of parameters, has been built at the working area already. According to analysis results of the relationship between landslide susceptibility and different geo-environment features, a logistic regression prediction model is established to calculate the landslide susceptibility of the whole county, which is divided into 17617 grid cells. The evaluation results reflect the characteristics of landslide disasters in Xinping County, and quantitatively provide the landslide probability of each cell, which is an important basis for landslide early warning. Then, the landslide warning thresholds and mufti-parameter comprehensive warning model was presented, which includes landslide susceptibility zonation, village maximum potentiality parameter, antecedent effective precipitation value and intraday induced precipitation value, antecedent effective precipitation and slope; etc. The results were used in the landslide disaster mitigation work during 2008-2010, and were proven to be effective.

Depth study on Multi-parameter prediction model for single landslide monitoring and forecasting was carried out by Deying Li[14]. Typical landslides with step-like deformation in the Three Gorges Reservoir are investigated based on site investigation and the analysis of monitoring data, involving The shape of displacement curve, deformation character, influencing factors and types of deformation and failure. Then the temparal and spatial deformation characteristics of landslide are investigated by means of the analysis of engineering geology condiction, deformation process, deformation mechanism and influencing factors. Based on the affecting factors of the water level and rainfall, the couping $\mathrm{GM}(1,1)$-BP model is established for landslide displacement prediction. At last, the prediction method of alert velocity is set up for the landslides prediction. A three-dimensional criteria of landslide prediction is proposed on the basis of the alert velocity, earth crack and macroscopical evidences.

\section{Conclusions}

At present, the majority of researchers agree the point of view as follows: combining the landslide deformation mechanism analysis and quantitative forecasting, using systematic integration and systematic analysis, is the only way to identify landslide and forecast accurately. Considering the case study and application effect, comprehensive forecasting method of landslide disasters is adopted. The basic ideas of this method, which including landslide deformation evolution research, correlation between external factors and sensitivity analysis, and temporal and spatial combination, combines the landslide formation mechanism and landslide monitoring and warning technology. The forecast 
criterion determining method based on comprehensive multifactor which includes establishing numerical simulation based on geological analysis, monitoring system simulation analysis, intelligent information technology can fully reflect the personality characteristics and deformation of landslide evolution, ensures maximum effectiveness of the monitoring and early warning. It is still difficult to carry out large-scale promotion because of the high demand for theory, technical and financial aspects, the above method is more suitable for important small area or single landslide's monitoring and early warning. We should believe it has broad application prospects. Furthmore, it can be provided reference for monitoring systems design of other geological disasters.

\section{Acknowledgements}

This work was financially supported by the Hebei Natural Science Foundation (E2013210023).

\section{References}

[1] Dayong Liu, Ende Wang, Jianchao Song and Chengshuai Zhang: Journal of Catastropholocy, Vol.23 (2008), p.50, “ In Chinese”.

[2] Zhilei Wang: Predictive method for landslide dynamic deformation affected sensitively by rainfall and research on the effect of drainage tunnel (PhD Dissertation, Zhejiang University ,2012) " In Chinese".

[3] Qiang Xu and Yuping Zeng: Chinese Journal of Rock Mechanics and Engineering Vol.28 (2009), p.1099, “ In Chinese”.

[4] Cong Li:Research on prediction and early warning of deformation and stability evolution of slopes (PhD Dissertation, China University of Geosciences. 2011) “ In Chinese”.

[5] Jiamei Xu: The Chinese Journal of Geological Disaster and Control Vol.16 (2005), p.152, “ In Chinese".

[6] Wu yi and zhaoping meng: Rock and Soil Mechanics Vol.28(2007), p.2529 “ In Chinese”.

[7] Miaoqian Wang, Mowen Xie and Man Hu: Science \& Technology Vision Vol.16 (2015), p.133, “ In Chinese".

[8] Kunlong Yin and Tongzhen Yan: Chinese Journal of Rock Mechanics and Engineering Vol.15 (1996), p.1, “ In Chinese”.

[9] Tianbin Li: The Chinese Journal of Geological Disaster and Control Vol.13 (2002), p.17, “ In Chinese".

[10] Xiuzhen Li,Qiang Xu,Runqiu Huang and Minggao Tang: The Chinese Journal of Geological Disaster and Control Vol.14 (2003), p. 5. “ In Chinese”.

[11] Shunmin Yi: Chinese Journal of Engineering Geophysics Vol.4 (2007), p.157, “ In Chinese”.

[12] Manchao He: Chinese Journal of Rock Mechanics and Engineering Vol.28 (2009), p.1081, “ In Chinese".

[13] Mingsheng Wen:Study on theory and method of rainfall-induced landslide early-warning in Ailao mountain(PhD Dissertation, Beijing Jiaotong University,2014), “ In Chinese”. 
[14] Deying Li:Prediction study of landslides with step-like deformation in the Three Gorges Reservior (PhD Dissertation, China University of Geosciences. 2010) “ In Chinese”. 